\title{
UDC highs and lows in Cornwall
}

\author{
By Sarah Mount
}

$\mathrm{n}$ behalf of my colleagues and I working at the NHS England approved Urgent Dental Care (UDC) Hubs in Bodmin and now Truro I thought it timely to share an insight into the highs and lows that we have encountered during the COVID-19 pandemic.

I am usually a paediatric dentist working primarily for the Community Dental Services in Cornwall plus two days a week in general practice. However, when faceto-face dentistry ceased, I became heavily involved in the frantic setting up of the UDC Hubs.

This can only be described as an emotional rollercoaster, both professionally and personally.

In our initial coordination efforts, consideration was given to rapidly changing guidelines and protocols, standard operating procedures, and accessibility of appropriately fitting PPE. The logistics were vast, complicated and have proven to be an ongoing, constantly evolving challenge with many extraordinarily frustrating delays. Our entire services were forced to either cease or quickly restructure to accommodate the triaging of patients and provision of remote teleconsultations to deliver the much talked about advice, analgesia and antimicrobials (AAA) towards arranging face-to-face consultations only for those who absolutely need this. At the heart of this planning, our focus was and has remained on maintaining the safety of our colleagues, their families, patients and the wider community, and to ensure that as dental professionals we are not vectors, facilitating the wider spread of the virus.

In the early days of planning the UDC Hub at Harleigh Road in Bodmin, a dentist colleague and I travelled to Exeter to be taught by accredited trainers in fit testing respirators, therefore becoming mask fit trainers ourselves. There were initial difficulties in receiving this fit test training as the local hospitals were rightfully prioritising intensive care and emergency departments. In reality fit testing masks to our colleagues was a considerable task. We had not anticipated that for every brand of FFP3 mask you are required to fit test each new mask. Coupled with difficulties in obtaining FFP3 masks in the first instance owing to the national shortage and the varying brands of masks being delivered by NHS Supply Chain, together with the inability to reorder the much needed Bitrix solution to facilitate mask testing, made this an endlessly demanding task. A further complication was the delivery of what appeared to be out of date/re-labelled
Once we had overcome the difficulties experienced in receiving the mask fit training and the intermittent supply of FFP3 masks, other PPE and the Bitrix solution, we subsequently trained our clinical colleagues but the opening of the UDC Hub was further delayed for numerous other reasons including complications in obtaining the appropriate clean down agents. Whilst we have continuously adapted our operations and identified different suppliers to address these challenges, there were various other factors to consider before opening including, as simple as it may sound, how the patient

\section{'The FFP3 masks are uncomfortable, cumbersome and induce a degree of claustrophobia'}

expiry dates on our much sought after FFP3 masks from NHS Supply Chain. We were later assured this PPE had passed stringent independent tests that demonstrate they are safe by the Health and Safety Executive (HSE) but this only serves to highlight the hurdles we're encountering.

The FFP3 masks themselves are nothing like the standard fluid-resistant surgical masks that we have all become accustomed to wearing in the dental surgery. They are uncomfortable, cumbersome and induce a degree of claustrophobia. Many wearers find skin irritation a common problem. A significant number of colleagues did not pass the fit test, primarily due to sizing. The masks so far are of standard sizes which do not necessarily fit all face shapes and sizes. One colleague had to shave off his beard in order to pass the fit testing that same day. Perhaps in the future we may be able to order smaller masks for smaller faces, but with the ongoing shortages this is currently an impossible hope. would enter the UDC Hub on arrival, maintaining social distance from other members of the general public. Another challenge was how to completely strip each surgery, as the entire room along with all equipment used needs to be thoroughly cleaned after each patient, imposing significant time restrictions and further limiting the number of patients we can see per day. We had several trial runs before we all felt comfortable and safe with our 'new normal'.

Finally, on 11 April 2020 we opened our UDC Hub in Bodmin to see patients face-to-face. This was ahead of schedule and it must be said that we worked tirelessly throughout the Easter weekend and have continued to do so, restricting appointments only when PPE levels have necessitated this. The Smile Together team has been incredibly supportive, agile and innovative, and we have rapidly adapted to our new working environment and use of different technologies with minimal fuss and 
4 maximum efficiency. I am grateful to work alongside such lovely caring colleagues who have shown up to work under what can only be described as difficult and unprecedented circumstances.

We are also indebted to my principal dentist and practice owner in general practice, Dr Steven Pace from Threemilestone Dental Practice, who has generously permitted me to dedicate my general practice days to the UDC Hub and ultimately benefit local patients.

In fact we've had tremendous support from the dental community during this unprecedented time and wanted nothing more than to announce that our first UDC Hub was open and shine a light on our collective contribution, but refrained to avoid inadvertently driving up patient demand by doing so.

We take into consideration each individual patient, their medical history and best interests, but treat according to guidance from NHS England. For the UDC Hub to run at maximum capacity for the highest level of emergencies only, our treatment modalities have primarily focussed on extracting teeth but we have also re-implanted several avulsed anterior teeth following trauma and extirpated when necessary. In such cases rubber dam is an absolute necessity and this is no mean feat for a child to tolerate whilst we are wearing enhanced levels of PPE which means our faces are completely covered and we must appear very intimidating. Equally our PPE, which encompasses the wearing of goggles, face shields, gowns etc, is cumbersome and incredibly hot and, as mentioned previously, the FFP3 masks are a challenge to work in. These factors coupled with the policy of not permitting fans or air conditioning in the surgery means that the working conditions are certainly less comfortable than what we are used to. Nevertheless, our outcomes have been impressive under such challenging circumstances.

The availability of PPE has been a constant problem for us all and this is reflected at a national level, particularly shortages of the specified FFP3 masks and fluid resistant long-sleeved gowns. As we're performing AGPs in our UDC Hubs we continue to operate with the highest level of PPE for which supplies have been inconsistent at best and our PPE stock levels have fluctuated, directly limiting the number of patients

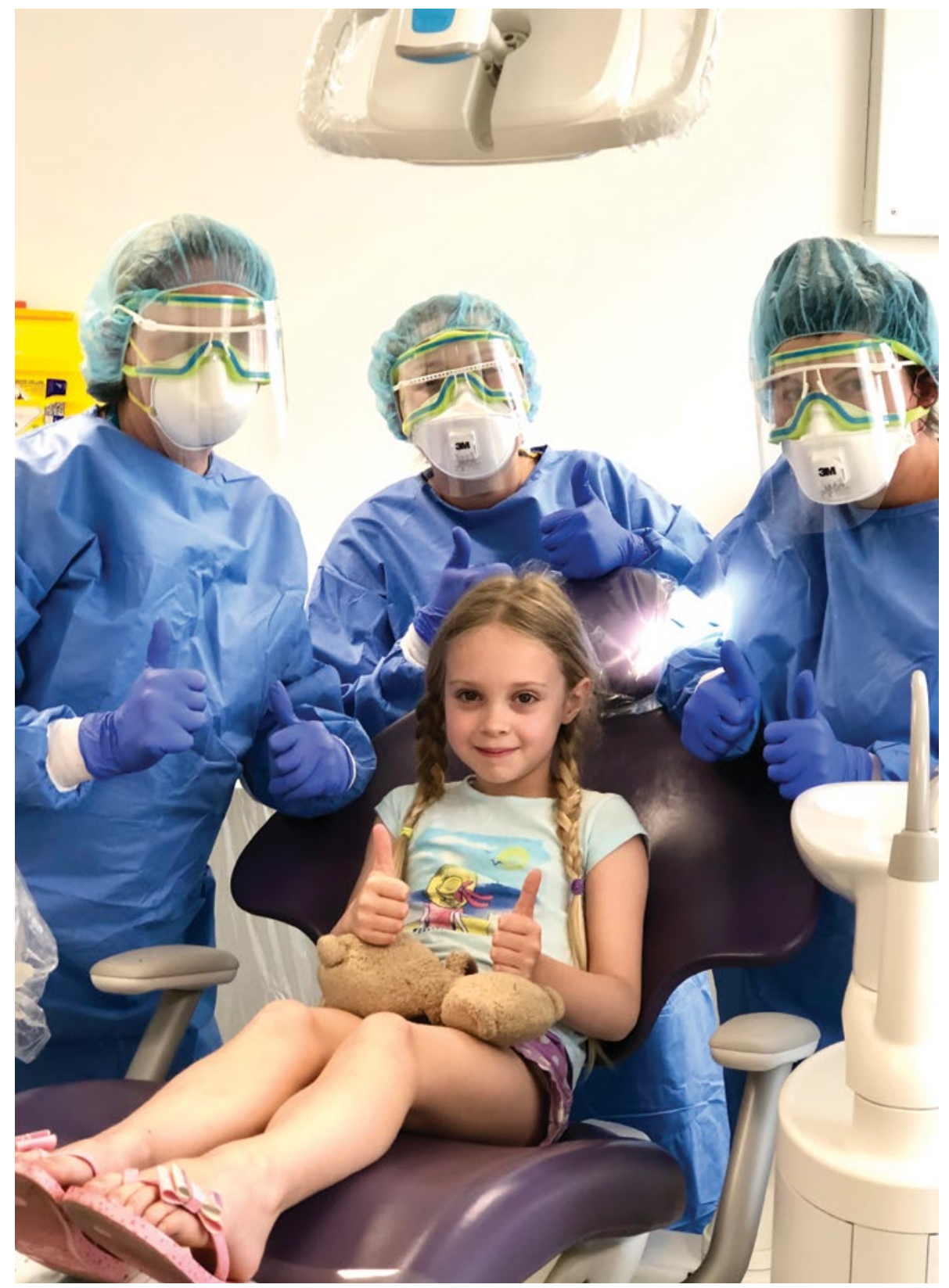

we can see. We often run critically low, necessitating strict inclusion of P1 criteria only and we can only apologise to those dentists referring in $\mathrm{P} 2 / 3$ cases and whom we have not been able to treat.

We are truly grateful to those suppliers who are doing their best to supply the required PPE without the profiteering we've seen from some and we very recently received a small number of reusable respirator masks from Royal Cornwall Hospital Trust (RCHT) and, once fit tested, hope they will prove helpful in preserving our precious FFP3 mask stocks. In the short term we anticipate PPE shortages will compound and get increasingly worse, particularly as more UDC Hubs open, but in the long term hope this situation will improve and enable a greater consistency of patient care.

In Cornwall, referrals for the UDC Hubs are sent directly to the Smile Together referral management team (of which I'm a part) via the online NHS triaging form. All incoming referrals are continuously monitored and managed throughout the day and regularly into the evening, seven days a week and always prioritising patients according to need to ensure we treat the most severe cases. During the normal working weekday, referrals are received from both GDPs in general practice who are triaging their own patients (and often unregistered ones too), together with our existing Access (Unscheduled Care) Services who are dealing primarily with a high proportion of 
\& unregistered patients. Over the weekends and inclusive of Bank Holidays our Out of Hours $(\mathrm{OOH})$ team has seen a significant surge in demand with the $\mathrm{OOH}$ dentists sending referrals into the Hub via the same process.

Prioritisation is based on classification $(\mathrm{P} 1 / 2 / 3)$ regardless as to whether they are registered or unregistered, NHS or private, as possible. For a few difficult cases we are thankful to have been able to directly liaise with our Oral Surgery Maxillofacial Consultants at RCHT for additional help and support.

It is fair to say that we have been inundated with referrals and there was a danger the Hub would be quickly overwhelmed, but the team have continued prioritising and treating

\section{'It's brought us all to tears on more than one occasion but we are beginning to smile more often!'}

and all referrals are triaged by dentists only. Once a referral has been received into the Hub, we liaise directly with the patient to confirm validity and assess for suitability. This includes obtaining photographs detailing the extent of swelling or video conferencing so that we have as much detail patients throughout. Initially, with only one Hub open at Bodmin and inconsistent PPE supplies, capacity remained critical. This has not hugely eased despite additional Hubs opening in recent weeks as PPE shortages remain a critical factor. The supply and demand equilibrium is not yet balanced in favour of treating as many patients as possible but as more UDC Hubs become operational we hope this will change.

We will continue to manage incoming referrals and provide treatment at the Hubs for as long as is necessary. We really are trying our hardest to accommodate as many patients as possible in what can only be described as unprecedented, tough and continually changing circumstances.

We will continue to evolve patient care and service provision across Cornwall and the Isles of Scilly as best as we can. It's brought us all to tears on more than one occasion but we are beginning to smile more often than we were!

Written in May 2020

The dentists delivering patient care face to face across Cornwall have been Sarah Mount, Rebecca Hammond, Assal Tabatabai, Florence Phillips, Clare Newland, Gill Clarkson (on the Isles of Scilly), Kannan Mathivanan, Brian Needham and Michael Cox. They have been supported by a large team of dental nurses, clinical team lead nurses, an Operations Team and Call Centre colleagues: a monumental team effort all round. 